\title{
The Possible Interpretations of Y(4260)
}

\author{
Shi-Lin $\mathrm{Zhu}^{1,2}$ \\ ${ }^{1}$ Department of Physics, Peking University, Beijing 100871, China \\ ${ }^{2} \mathrm{RCNP}$, Osaka University, Japan
}

October 29, 2018

\begin{abstract}
The recently observed Y(4260) lies far above the decay threshold with a width less than $100 \mathrm{MeV}$. We argue that it's very difficult to accommodate Y(4260) as a conventional $c \bar{c}$ radial excitation or a D-wave state. It can't be a hadronic molecule. Its production mechanism and special decay pattern do not favor the glueball interpretation. If $\mathrm{Y}(4260)$ is a scalar tetraquark, it must be produced by the $I=0$ component of the virtual photon. Then the $I=1, I_{z}=0$ component of the virtual photon should have produced its isovector partner $Y^{\prime}(4260)$, which may be searched for in the decay channel $\pi^{+} \pi^{-} \pi^{0} J / \psi$ using exactly the same database from the initial state radiation process. The observation/non-observation of $Y^{\prime}(4260)$ can easily confirm/reject the tetraquark hypothesis. However, a tetraquark far above threshold can fall apart into $D \bar{D}, D^{*} \bar{D}$ very easily. Its not-so-large width and the non-observation of $D \bar{D}$ mode tend to disfavor the tetraquark hypothesis. Hence the only feasible interpretation is a hybrid charmonium if $\mathrm{Y}(4260)$ is NOT an experimental artifact. At present, none of the experimental information from BABAR measurement is in conflict with the hybrid charmonium picture.
\end{abstract}

PACS number: 12.39.-x, 13.20.Gd, 13.25.Gv, 14.40.Gx

\section{Introduction}

The past several years have witnessed a revival of interest in hadron spectroscopy due to the dramatic experimental progress. Many new narrow resonances were observed, most of which lie close to the threshold. Among them, we have $D_{s j}(2317)[1], D_{s j}(2460)[2]$, $D_{s j}(2632)[3], \Theta^{+}[4], \mathrm{X}(3872)[5], \mathrm{Y}(3940)[6]$.

Now the pentaquark legend is fading [7]. $D_{s j}(2632)$ was not confirmed by other groups. One may wonder whether it's an experimental artifact [8]. Luckily $D_{s j}(2317), D_{s j}(2460)$ and $\mathrm{X}(3872)$ were confirmed by other collaborations and survived as well-established resonances. It's plausible that $D_{s j}(2317)$ and $D_{s j}(2460)$ are the conventional $\left(0^{+}, 1^{+}\right)$ doublet in the heavy quark effective field theory while their masses are lowered through their coupling to the nearby $D K$ and $D^{*} K$ thresholds [9]. 
The hidden charm state $\mathrm{X}(3872)$ remains mysterious. According to Ref. [10], the conventional $1^{++}$quark model lies several tens $\mathrm{MeV}$ higher than $\mathrm{X}(3872)$. In Ref. [11] the authors used a potential model to include the coupled-channel effects the open charm threshold which increases the "bare" $2^{3} P_{1}$ state by $30 \mathrm{MeV}$. The previously prevailing molecule picture [12] encountered serious challenges recently. The molecule picture predicted a tiny branching ratio for the decay mode $X(3872) \rightarrow D \bar{D} \pi$, which is two orders of magnitude smaller than the recent experimental data $[13,14]$. Moreover, the molecule picture predicted the ratio between $B^{0} \rightarrow X(3872) K^{0}$ and $B^{+} \rightarrow X(3872) K^{+}$to be much less than $10 \%$ [15] while the experimental data is order unity, $0.61 \pm 0.36 \pm 0.06$ [14]. The large branching ratio of $D \bar{D} \pi$ mode indicates that the core of $\mathrm{X}(3872)$ is probably the conventional $1^{++}$quark model state, which is only several tens of $\mathrm{MeV}$ higher than the $D \bar{D}^{*}, \rho J / \psi, \omega J / \psi$ thresholds. There may exist strong coupling between the bare quark model state and the continuum, which lowers the $1^{++}$bare state and transforms this state into $\mathrm{X}(3872)$. In other words, $\mathrm{X}(3872)$ is a mixture of the dominant $1^{++}$bare $c \bar{c}$ state and a small portion of $D \bar{D}^{*}, \rho J / \psi, \omega J / \psi$ continuum. Similar views can be found in [16]. Phenomenology based on this new picture is still lacking.

Very recently BABAR collaboration observed a broad resonance around $4.26 \mathrm{GeV}$ in the $\pi^{+} \pi^{-} J / \psi$ channel [17]. Its decay width is around $(50 \sim 90) \mathrm{MeV}$. A remarkable feature of this state is that it lies far above threshold but still has a rather narrow width.

Up to now, there are $125 \pm 23$ total events [17]. Since this resonance is found in the $e^{+} e^{-}$annihilation through initial state radiation $e^{+} e^{-} \rightarrow \gamma_{I S R} Y(4260)$, its spin-parity is determined to be $J^{P C}=1^{--}$. However, this state is not observed in the direct search $e^{+} e^{-} \rightarrow Y(4260)$ because of its low ratio $0.34 \%$, buried by the $4 \%$ experimental uncertainty for the hadronic cross section [17]. The non-observation strongly indicates that $Y(4260) \rightarrow \pi^{+} \pi^{-} J / \psi$ is one of the dominant decay modes. At least the branching ratio of this mode should be much larger than that of $D \bar{D}$. Otherwise the latter mode should have been observed [17].

\section{Possible Interpretations}

If $\mathrm{Y}(4260)$ really exists, the available experimental data contains useful information on its underlying structure. In fact, BABAR may have found the elusive hybrid charmonium. We shall analyze various possibilities one by one, assuming $\mathrm{Y}(4260)$ is a genuine resonance and not an experimental artifact.

\subsection{Conventional $c \bar{c}$ State}

With $J^{P C}=1^{--}$, a conventional $c \bar{c}$ state is either a radial excitation or a $\mathrm{D}$ wave state. From PDG [18], the masses of the well-established radial excitations: $\psi(2 S), \psi(3 S), \psi(4 S)$ are $3686 \mathrm{MeV}, 4040 \mathrm{MeV}, 4415 \mathrm{MeV}$ respectively. The masses of $\psi\left(1^{3} D_{1}\right)$ and $\psi\left(2^{3} D_{1}\right)$ are $3770 \mathrm{MeV}$ and $(4159 \pm 20) \mathrm{MeV}[18]$. The $\psi\left(3^{3} D_{1}\right)$ state should lie above $4.5 \mathrm{GeV}$. Therefore, it's nearly impossible to accommodate Y(4260) as a conventional charmonium radial excitation or D-wave state. 


\subsection{Couple-Channel Effects}

One may wonder whether Y(4260) may arise from some couple-channel effects. I.e., the bare charmonium state couples strongly with the continuum. In this way, the bare quark model mass is shifted below while its wave function admits some contribution from the continuum as in the case of $D_{s j}(2317,2460)$ and X(3872).

Among all the known cases where the couple-channel effect plays an important role, there is a common feature. The resonance lies very close to the decay threshold. A well-known example is the $f_{0} / a_{0}(980)$, which is within $10 \mathrm{MeV}$ of $K \bar{K}$ threshold. The recent X(3873) sits nearly exactly on the threshold. $D_{s j}(2317,2460)$ lies also very close to $D K, D^{*} K$ threshold. Quantum mechanics tells us that the mixing strength is related to the inverse of the energy difference of the two states.

$\mathrm{Y}(4260)$ is far away from both the open-charm and hidden charm decay threshold. The analysis in Ref. [11] shows that the mass shift of the $c \bar{c}$ state caused by the coupled-channel effects of the open charm thresholds is only around tens MeV. If the PDG charmonium assignments are correct, it's very difficult to shift the mass of the $3^{3} D_{1}$ state from above 4.5 GeV down to $4.26 \mathrm{GeV}$ by the coupled-channel effects.

\subsection{Molecule}

Hadronic molecules are states which lie below the continuum threshold and carry a small amount of binding energy. $\mathrm{Y}(4260)$ lies far above the $D \bar{D}, \omega J / \psi$ threshold.

One may wonder whether $\mathrm{Y}(4260)$ is a hadronic molecule composed of $\bar{D}_{s} D_{s j}(2317)$ since it is only $26 \mathrm{MeV}$ below $\bar{D}_{s} D_{s j}(2317)$ threshold. However the angular momentum and parity conservation exclude the possibility of $\bar{D}_{s} D_{s j}(2317)$ forming a $1^{--}$molecular state.

$\mathrm{Y}(4260)$ is $24 \mathrm{MeV}$ below $\bar{D} D_{1}(2420)$ threshold. $D_{1}(2420)$ is a very narrow resonance. Its total width of $(50 \sim 90) \mathrm{MeV}$ disfavors the identification as a $\bar{D} D_{1}(2420)$ molecular state. The total width also disfavors the identification as a $\bar{D} D_{1}^{\prime}$ or $\bar{D}_{0}(2310) \bar{D}^{*}$ or $\bar{D}^{*} D_{1}^{\prime}$ molecule since both $D_{0}(2310)$ and $D_{1}^{\prime}$ are very broad resonances. Hence Y(4260) can not be a hadronic molecule.

\subsection{Glueball}

C parity requires that a $J^{P C}=1^{--}$glueball contains at least three gluons. The decay patterns of the glueballs are expected to be flavor-blind. If it is a glueball, $\mathrm{Y}(4260)$ should decay into multiple light mesons very easily with so large phase space. Certainly $\pi^{+} \pi^{-} J / \psi$ can not be a dominant decay mode. On the other hand, Y(4260) is produced through initial state radiation. The photon does not couple to gluons. Hence the possibility of $\mathrm{Y}(4260)$ being a glueball is tiny.

\subsection{Tetraquark}

Naively one may speculate $\mathrm{Y}(4260)$ could be a tetraquark with the quark content $\frac{1}{\sqrt{2}}(u \bar{u}+$ $d \bar{d}) c \bar{c}$. In fact the presence of a heavy quark pair favors the formation of a tetraquark 
since the heavy quark pair lower the kinetic energy significantly.

With $J^{P C}=1^{--}$, one orbital excitation is required for a tetraquark. Hence its mass is roughly

$$
M_{Y}=2\left(m_{c}+m_{u}\right)+\Delta E_{L}
$$

where $m_{c}, m_{u}$ is the charm and up quark constituent mass, $\Delta E_{L}$ is the orbital excitation energy. With $m_{c}=1.63 \mathrm{GeV}, \Delta E_{L}=(0.30 \sim 0.45) \mathrm{GeV}[10], m_{u}=0.33 \mathrm{GeV}$, the mass of this tetraquark is roughly $(4.22 \sim 4.37) \mathrm{GeV}$, very close to the experimental value 4.26 $\mathrm{GeV}$. The above discussion shows the attractive side of the tetraquark hypothesis.

However, this hypothesis faces one serious obstacle: the not-so-large decay width measured by BABAR Collaboration [17], $\Gamma_{Y}=(50 \sim 90) \mathrm{MeV}$. If $\mathrm{Y}(4260)$ is a scalar tetraquark with the quark content $\frac{1}{\sqrt{2}}(u \bar{u}+d \bar{d}) c \bar{c}$, it can easily decay into $D \bar{D}$ final states via P-wave. Such a decay occurs through the so-called "super-allowed" "fallapart" mechanism, which is simply the regrouping of the two quarks and anti-quarks inside the tetraquark into two color-singlet mesons. No symmetry forbids the decay of $Y(4260) \rightarrow D \bar{D}$. With enough phase space, one would expect its total decay width to be around several hundred $\mathrm{MeV}$ or more, contrary to the experimental value $(50 \sim 90)$ $\mathrm{MeV}$. Moreover, $D \bar{D}$ should be one of the dominant decay modes, which is also against BABAR's observation.

In fact, BABAR Collaboration can further confirm/reject the tetraquark conjecture through the observation/non-observation of the isovector tetraquark $Y^{\prime}(4260)$ in the $\pi^{+} \pi^{-} \pi^{0} J / \psi$ channel by analyzing the same set of initial state radiation data.

If $Y(4260)$ is a tetraquark, it must belong to a $S U_{F}(3)$ flavor multiplet. In the present case, we have a nonet from $3_{F} \times \overline{3}_{F}=1_{F}+8_{F}$. In the vector channel, the flavor eigenstates always split into the mass eigenstates because of the ideal mixing. A typical example is that the physical states are $\omega$ and $\phi$ instead of $\omega_{0}$ and $\omega_{8}$. We list the quark content of these nine states below: (1) $\mathrm{I}=0, \mathrm{Y}(4260), \frac{1}{\sqrt{2}}(u \bar{u}+d \bar{d}) c \bar{c}$; (2) $\mathrm{I}=0, Y_{s s}(4560), s \bar{s} c \bar{c} ;(3)$ $I=1, I_{z}=0, Y^{\prime}(4260), \frac{1}{\sqrt{2}}(u \bar{u}-d \bar{d}) c \bar{c} ;(4) I=1, I_{z}= \pm 1, Y^{ \pm}(4260), u \bar{d} c \bar{c}, d \bar{u} c \bar{c} ;(5)$ $I=\frac{1}{2}, I_{z}= \pm \frac{1}{2}, Y_{s}^{\prime}(4410), u \bar{s} c \bar{c}, d \bar{s} c \bar{c}, s \bar{d} c \bar{c}, s \bar{u} c \bar{c}$.

The neutral isovector state $Y^{\prime}(4260)$ deserves special attention. With $J^{P C}=1^{--}$and $I=1$, its G-parity is positive. While $J / \psi$ carries negative G-parity, the conservation of G-parity demands $Y^{\prime}(4260)$ decays into three or five or seven pions.

The isospin of the virtual photon is indefinite. It contains both $I=1, I_{z}=0$ and $I=0, I_{z}=0$ components. In the extreme case that $\mathrm{Y}(4260)$ really turns out to be an isoscalar tetraquark, it must have been produced by the $I=0, I_{z}=0$ component of the virtual photon in the $e^{+} e^{-}$annihilation through initial state radiation. Then the accompanying isovector tetraquark $Y^{\prime}(4260)$ should also have been produced by the $I=1, I_{z}=0$ component of the virtual photon with the same ratio. BABAR Collaboration may search the $\pi^{+} \pi^{-} \pi^{0} J / \psi$ channel to look for the possible $Y^{\prime}(4260)$ signal in their initial state radiation data.

Moreover we urge BABAR Collaboration to scan their data to search for the possible $Y_{s s}(4560)$ signal in the $K \bar{K} J / \psi$ final states. It will also be useful to study the angular distribution to find out whether the decay proceeds via S-wave.

In short, more experimental investigation is needed to completely exclude the possibility of $Y(4260)$ being a tetraquark although the available data already disfavors the 
tetraquark hypothesis.

\subsection{Hybrid Charmonium}

Naively when one gluon is confined within a hadron bag, its binding energy is roughly around $1.1 \mathrm{GeV}$. Hence the $J^{P C}=1^{--}$hybrid charmonium mass may be around $4.2 \mathrm{GeV}$. Some lattice QCD calculation found that the exotic $1^{-+}$hybrid charmonium lies around $4.3 \mathrm{GeV}[19,20]$. There is no lattice QCD calculation of $1^{--}$hybrid charmonium mass.

According to QCD sum rule calculation [22] and the analysis in the flux tube model [23], a hybrid meson tends to decay into final states with one $\mathrm{L}=1$ meson. An extensive study of open charm decay modes can be found in Ref. [24]. It is very interesting to note that these author did predict a small $D \bar{D}$ width and a rather narrow $1^{--}$hybrid charmonium if its mass is below $4.3 \mathrm{GeV}$. BABAR may search the other decay modes as suggested in [24].

There is not much discussion of the hidden charm decay modes of the hybrid charmonium in literature. Physically these decays may occur via the following process: $c \bar{c} G \rightarrow J / \psi+G G \rightarrow J / \psi+\pi^{+} \pi^{-}$. Because of the isospin symmetry, Y(4260) also decays into $\pi^{0} \pi^{0} J / \psi, \eta \eta J / \psi$. Motivated by the discovery mode, we suggest the decay modes $Y(4260) \rightarrow \omega+\chi_{c 0, c 1, c 2} \rightarrow 3 \pi+\chi_{c 0, c 1, c 2}$ may be important if Y(4260) is really a hybrid meson.

\section{Discussion}

We have excluded the possibility of $\mathrm{Y}(4260)$ being a conventional $c \bar{c}$ state, a molecule, a glueball one by one using the available experimental information. The tetraquark hypothesis is not favored by its small total width and non-observation of $D \bar{D}$ decay mode. In order to completely exclude this possibility, BABAR may search for its isovector partner using the same database in the $\pi^{+} \pi^{-} \pi^{0} J / \psi$ channel. The most plausible interpretation is a hybrid charmonium, which is consistent with all the experimental information.

No matter whether $\mathrm{Y}(4260)$ is a tetraquark state or hybrid charmonium, it shall decay into $\pi^{0} \pi^{0} \mathrm{~J} / \psi$ if it's a genuine resonance. This branching ratio is $25 \%$ of the discovery mode from isospin symmetry consideration. The other possible mode is $\eta \eta J / \psi$, which may be suppressed by phase space. The electromagnetic decay modes $Y(4260) \rightarrow \gamma+$ $\chi_{c 0, c 1, c 2}$ are also very interesting. Confirmation of $\mathrm{Y}(4260)$ in a different channel and by a different experimental group in the near future will greatly shed light on the charmonium spectroscopy.

\section{Acknowledgment}

This project was supported by the National Natural Science Foundation of China under Grants 10375003 and 10421003, Ministry of Education of China, FANEDD, Key Grant Project of Ministry of Education (NO 305001) and SRF for ROCS, SEM. 


\section{References}

[1] B. Aubert et al. (BABAR Collaboration), Phys. Rev. Lett. 90, 242001 (2003).

[2] D. Besson et al. (CLEO Collaboration), Aip Conf.Proc. 698, 497 (2004); Phys. Rev. D 68, 032002 (2003).

[3] A.V. Evdokimov et al. (SELEX Collaboration), Phys. Rev. Lett. 93, 242001 (2004).

[4] T. Nakano et al. (LEPS Collaboration), Phys. Rev. Lett. 91, 012002 (2003).

[5] S. K. Choi et al. (Belle Collaboration), Phys. Rev. Lett. 91, 262001 (2003).

[6] S. K. Choi et al. (Belle Collaboration), Phys. Rev. Lett. 94, 182002 (2005).

[7] Shi-Lin Zhu, Int. J. Mod. Phys. A 19, 3439 (2004); A 20, 1548 (2005).

[8] Y. B. Dai, C. Liu, Y. R. Liu, Shi-Lin Zhu, JHEP 0411, 043 (2004); Y.R. Liu, Shi-Lin Zhu, Y.B. Dai, C. Liu, Phys. Rev. D 70, 094009 (2004).

[9] Y. B. Dai, C. S. Huang, C. Liu, and Shi-Lin Zhu, Phys. Rev. D 68, 114011 (2003).

[10] T. Barnes, S. Godfrey, and E. Swanson, hep-ph/0505002.

[11] E. J. Eichten, K. Lane, and C. Quigg, Phys. Rev. D 69, 094019 (2004).

[12] E. S. Swanson, Phys. Lett. B 588, 189 (2004); 598, 197 (2004).

[13] G. Bauer, hep-ex/0505083.

[14] D. Bernard, Plenary talk at the International Conference on QCD and Hadronic Physics, June 16- 20, 2005, Beijing, China; http://www.phy.pku.edu.cn/ qcd/transparency/17-plen/Bernard.pdf

[15] E. Braaten and M. Kusunoki, Phys. Rev. D 71, 074005 (2005).

[16] C. Meng, Y. J. Gao, K.-T. Chao, hep-ph/0506222.

[17] B. Aubert et al. (BABAR Collaboration), hep-ex/0506081.

[18] Particle Dada Group, S. Eidelman et al., Phys. Lett. B 592, 1 (2004).

[19] X. Liao and T. Manke, hep-lat/0210030.

[20] Z. H. Mei and X. Q. Luo, Int. J. Mod. Phys. A 18, 5713 (2003).

[21] G. S. Bali, Eur. Phys. J. A 19, 1 (2004).

[22] Shi-Lin Zhu, Phys. Rev. D 60, 014008 (1999); D 60, 097502 (1999).

[23] F. E. Close and P. R. Page, Nucl. Phys. B 443, 233 (1995); F. E. Close and S. Godfrey, Phys. Lett. B 574, 210 (2003).

[24] P. R. Page, E.S. Swanson, A.P. Szczepaniak, Phys. Rev. D 59, 034016 (1999). 